\title{
Measurements of cervical range of motion using an optical motion capture system: Repeatability and validity
}

\author{
MINSHAN FENG ${ }^{1,2^{*}}$, LONG LIANG $^{1 *}$, WU SUN $^{1}$, GUANG WEI LIU ${ }^{2}$, \\ XUNLU YIN ${ }^{1}$, TAO HAN ${ }^{1}, \mathrm{XU} \mathrm{WEI}^{1,2}$ and $\mathrm{LIGUO} \mathrm{ZHU}^{1,2}$ \\ ${ }^{1}$ Department of Spine; ${ }^{2}$ Beijing Key Laboratory of Orthopedics of Traditional Chinese Medicine, \\ Wangjing Hospital of Chinese Academy of Chinese Medical Sciences, Beijing 100102, P.R. China
}

Received September 26, 2018; Accepted May 16, 2019

DOI: $10.3892 /$ etm.2019.8105

\begin{abstract}
The current study aimed to assess the repeatability and validity of cervical range of motion (CROM) measurements using an optical motion capture system (OMCS), compared with a CROM device. A total of 20 healthy volunteers were selected and enrolled in the current study after informed consent was received. The motion of the cervical spine in all directions was measured using the OMCS and CROM devices. Reproducibility of data was assessed using the intra-group correlation coefficient (ICC), standard error of measurement (SEM) and minimum detectable change (MDC). Validity was assessed using the coefficient of determination $\left(\mathrm{R}^{2}\right)$ in combination with Pearson's correlation coefficient. Bland-Altman plot were presented for the two measurement methods. The range of motion (ROM) was measured by using the OMCS and the CROM device during the same session. Both procedures evidenced high ICCs [OMCS: ICC $(1,2)$ $=0.802-0.981$; CROM device: $\operatorname{ICC}(1,2)=0.768-0.948]$, low SEM values (OMCS: $0.98^{\circ}-1.38^{\circ}$; CROM device: $1.04^{\circ}-2.45^{\circ}$ ) and low MDC values (OMCS: $2.72^{\circ}-3.81^{\circ}$; CROM device: $\left.2.89^{\circ}-6.78^{\circ}\right)$. A high $\mathrm{R}^{2}(0.568-0.882)$ and Pearson's correlation coefficient (0.753-0.939) were determined. The Bland-Altman plots demonstrated that most of the data were within the $95 \%$ consistency limit. In summary, the OMCS has good repeatability and validity when measuring CROM and is an effective way to evaluate cervical vertebral range of motion.
\end{abstract}

Correspondence to: Professor Liguo Zhu, Department of Spine, Wangjing Hospital of Chinese Academy of Chinese Medical Sciences, 6 Huajiadi Street, Beijing 100102, P.R. China

E-mail: 1071794037@qq.com

*Contributed equally

Key words: cervical range of motion, optical motion capture system, repeatability, validity

\section{Introduction}

Recent changes in society and lifestyle have caused a variety of cervical spine diseases to become more prevalent (1). A previous study demonstrated that neck pain accounts for $14.6 \%$ of all musculoskeletal diseases (2). Cervical diseases are common in individuals that have long-term immobilization of the neck or excessive neck activity, with most patients exhibiting flexor head injuries (3). A variety of cervical diseases lead to a decrease in cervical spine range of motion ROM $(1,4)$. Cervical ROM is an indicator of cervical pain and a variety of cervical spine diseases (2). ROM can be used as an evaluation parameter to monitor the effect of cervical disease treatment, including when assessing clinical results after anterior decompression and cervical spine fusion $(2,5,6)$. With ROM measurements, clinicians can adjust treatment plans according to cervical vertebral mobility changes (5). Accurate cervical ROM measurements are important. However, due to the complex anatomy of the cervical spine and the coupled movement of the cervical and thoracic spine, it is currently challenging to obtain accurate cervical ROM measurements $(6,7)$. Several methods are currently used to measure cervical vertebrae activity, including eye measurements $(8,9)$, general protractor use $(10)$, and cervical ROM (CROM) device $(3,11,12)$, cervical laser (13), radiographic (14), computed tomographic (15) and tap measurements $(10,16,17)$. Currently, no absolute method has yet been established for measuring cervical vertebral activity.

A CROM device consists of a plastic frame and three independent inclinometers. During use, the plastic frame is placed on the head of the patient to stabilize the device. Three independent inclinometers are attached to the frame in the sagittal, frontal and horizontal planes to indicate the position of the head during flexion and extension, lateral bending and cervical spine rotation $(3,11)$. Previous studies have reported a high validity and repeatability when using a CROM device $(2,18,19)$. Although this device has numerous advantages, it includes trunk motion and therefore does not measure pure cervical ROM.

The optical motion capture system (OMCS) is commonly used in biomechanical studies to measure human kinematics at high accuracy (20). The aim of the present study was to evaluate the test-retest reliability and validity of using OMCS and CROM, by comparing neck ROMs during flexion, extension, left lateral bending, right lateral bending, left rotation and right rotation. 


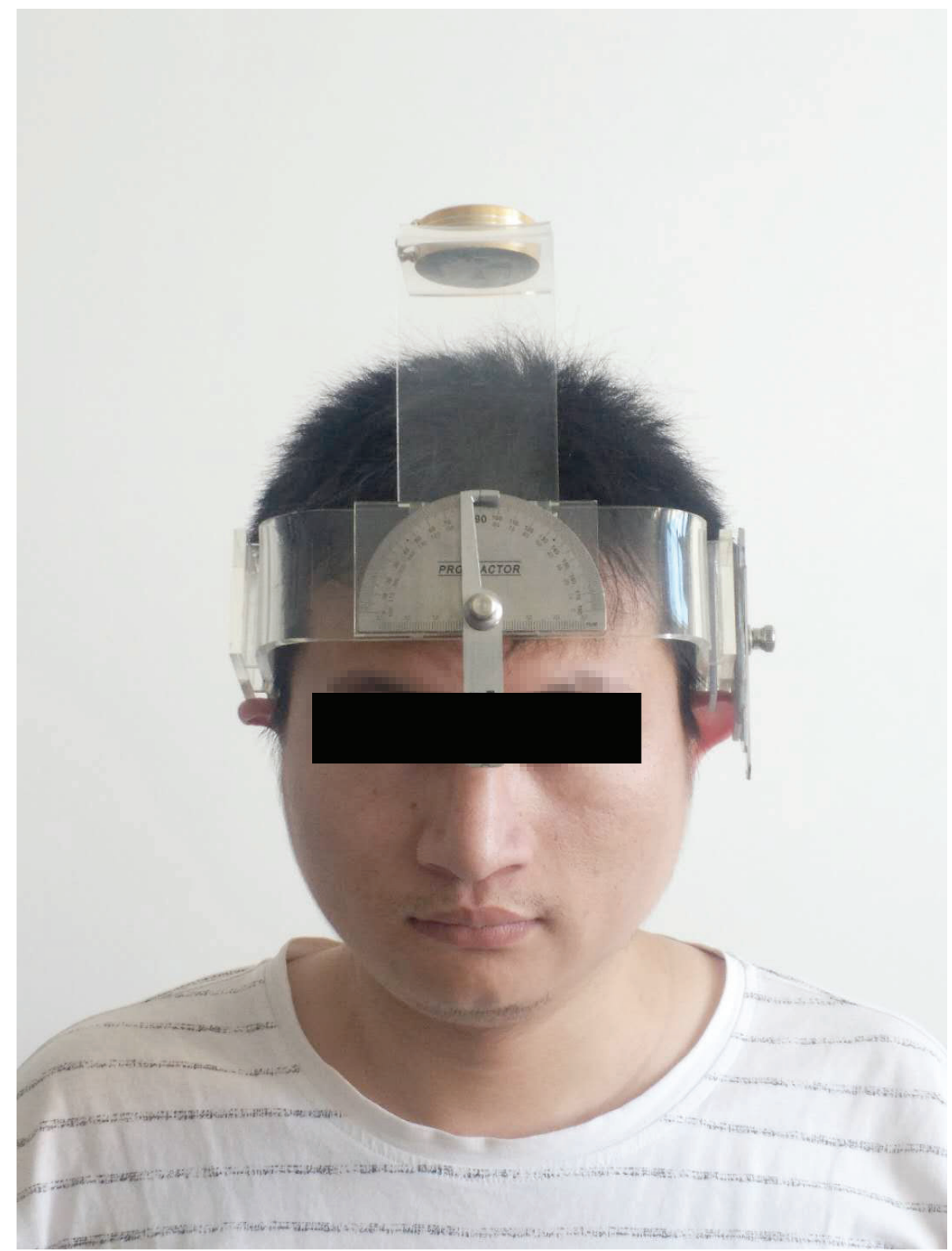

Figure 1. Participant wearing CROM device, which was placed on the upper edge of the eyebrow arch, with the lower back edge of the CROM device at the same level.

\section{Materials and methods}

Participants. From February 2018 to April 2018, a total of 20 healthy postgraduates (13 men; 7 women; mean age, $26.8 \pm 1.32$ years) from the Chinese Academy of Chinese Medical Sciences (Beijing, China) were included in the current study. The inclusion criteria were as follows: No cervical disease, no history of neck pain or limitation of cervical spine mobility, no bone, muscle or nervous system diseases and no history of neck surgery. The exclusion criterion was a history of neck disease or neck surgery. Prior to enrollment, each participant was screened for neck movement by a high-grade physician to determine if cervical vertebral activity was normal. The current study was approved by the Ethics Committee of Wangjing Hospital of Chinese Academy of Chinese Medical Sciences (Beijing, China) and each participant provided verbal and written informed consent prior to enrollment in the current study.
Instruments. Cervical spine ROM was measured using a CROM and OMCS device (V120:Trio; NaturalPoint, Inc.). Each measurement was recorded twice for each participant, with a 1-h interval.

CROM device measurement. The CROM device (Fig. 1) was placed on the participant's head while they were seated and looking directly forwards. The lower front edge of the device was placed on the upper edge of the eyebrow arch, with the lower back edge of the CROM device at the same level. The physician assisted in keeping the body (excluding the neck) fixed in position when movement was assessed by placing their hands on the participants shoulders. The difference between the pointer value and the value after movement was recorded as the motion angle of the cervical vertebrae. When measuring axis activity, the other two axis active pointers remained unchanged to avoid coupling motions in multiple directions. Two physicians viewed the degree of change exhibited by the 
CROM device to reduce subjective error. i) To test for neck flexion and extension, the chin and head was moved up and down. Each movement was stopped when the maximum angle was reached. The difference between the values of flexion limit, extension movement angle and the initial pointer value was used as the angle of activity. ii) To test for left and right neck flexion, participants flexed the neck to the left and right sides. When the maximum angle was reached, the movement was stopped and the change in the forehead protractor pointer was recorded. iii) To test for left and right neck rotation, participants rotated the neck to the left and right sides. When the maximum angle was reached, the movement was stopped and changes in the head pointer was recorded.

OMCS measurement. An OptiTrack-V120: Trio device (NaturalPoint, Inc.) was used to measure OMCS (Fig. 2). Optitrack Motive and Visual3D V5 software (NaturalPoint, Inc.) was used to visualize results. The OptiTrack-V120: Trio device was fixed in place with a 3-foot frame to ensure the cameras were in a horizontal position. This was located $2 \mathrm{~m}$ from the participant and at a height that was the same as that of the participants neck. All participants wore a motion capture suit (NaturalPoint, Inc.), which enabled the device to record measurements more accurately. Three marker points were placed at each of four positions: The forehead, the two bilateral temporal sites and at the top of the head. These were used to determine the position of the head when the body was rigid. No marker points were blocked or lost during the measurement process. Three additional marker points were located on both sides of the shoulder and the middle of the sternum. These were used to determine the chest position when the body was rigid. At least six marker points were located on the torso. The OptiTrack-V120 device was connected to the power supply and to the computer with the Optitrack Motive software via a USB connection line. The Optitrack Motive software was opened.

Participants were seated close to the back of the chair and looked straight ahead. Six movements of the cervical vertebrae were measured, including flexion-return, extension-return, left lateral bending-return, right lateral bending-return, left rotation-return and right rotation-return. During the measurements, the position of the head marker point was adjusted as required to avoid the marker points becoming blocked. The movement should be slow and smooth. The outer limit position of each action should remain for 1-2 sec to facilitate the determination of each position in the analysis process. Optitrack Motive software tracked marker point movement as presented in Fig. 3. Dynamic images of cervical spine mobility measurements in all directions are shown in Videos S1-6.

Tracked movement recorded by the software was saved as a C3D file and imported into Visual3D software. Angles were then calculated as presented in Fig. 4.

The measurement process of this experiment is that two instruments measure cervical range of motion at the same time, and the physician assisted in keeping the body (excluding the neck) in a fixed position, as presented in Fig. 5.

Statistics. Data were analyzed using SPSS version 22 software (IBM Corp.) and presented as the mean \pm standard deviation (SD). Test-retest repeatability was evaluated using the mean of the first and second measurements and the inter-class correla-

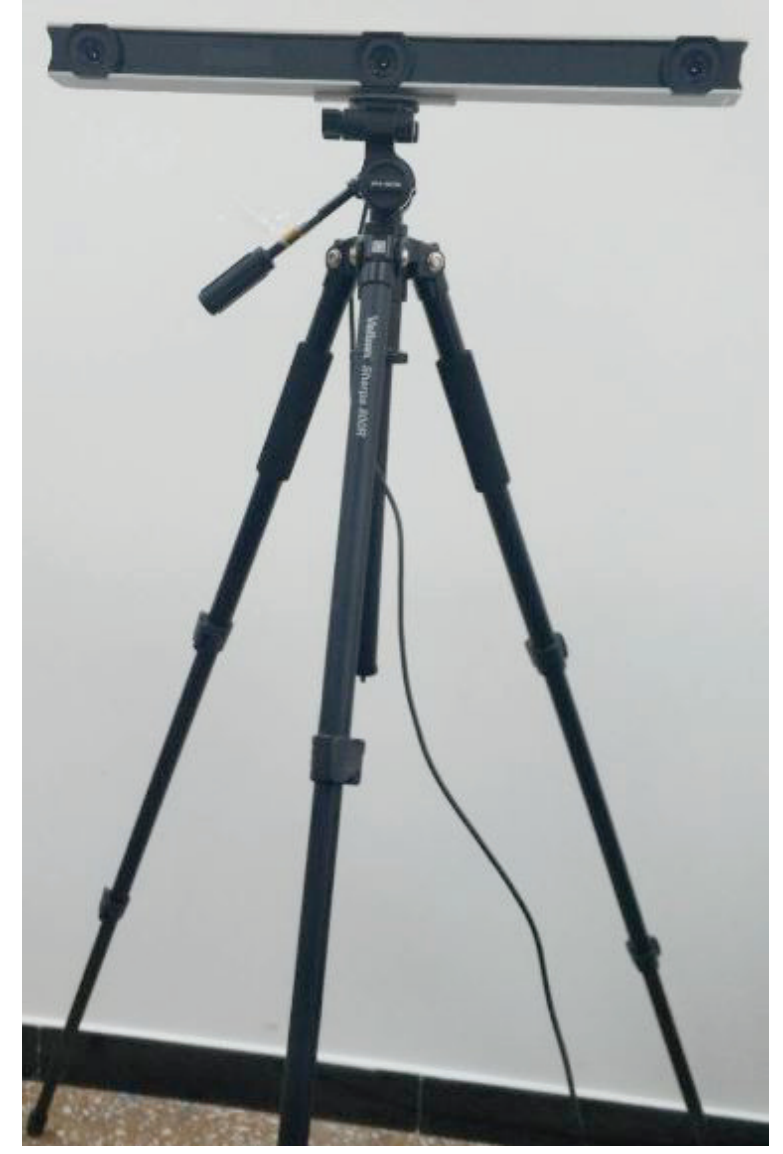

Figure 2. OptiTrack-V120: Trio device which represents the optical motion capture system. The device was fixed with a 3-foot frame to ensure that the three cameras were placed in a horizontal position.

tion coefficient (ICC), which describes one-rater (intra-rater) reliability of the two trials. The absolute reliability of the measurement was evaluated using the SEM and the minimum detectable change (MDC). The SEM was calculated using the following formula (21): $\mathrm{SEM}=\mathrm{SD} \times \sqrt{1-\mathrm{ICC}}$. The $95 \%$ confidence interval (CI) of the MDC was subsequently calculated based on the SEM value (22). MDC was calculated using the following formula: $\mathrm{MDC}=\mathrm{SEM} \times \sqrt{2} \mathrm{x} \sigma 95$, where the б95 score was the $95 \%$ CI of 1.96. ICC values for intra-rater agreement were interpreted as: 0-0.2 (poor), 0.2-0.4 (fair), 0.4-0.6 (moderate), 0.6-0.8 (substantial) and 0.8-1.0 (almost perfect) (23). ICC $\geq 0.6$ was considered to represent substantial reliability (24).

To check data validity, a linear regression analysis was performed for the CROM measurements recorded by the $\mathrm{CROM}$ and OMCS devices. Mean differences in the measured data from the two methods were calculated and scatter plots, regression lines, the determination coefficient $\left(\mathrm{R}^{2}\right)$ and Pearson's correlation coefficient were used to support method validity. Bland-Altman plots were also used to display the differences between the outcomes of the two methods (25).

\section{Results}

Repeatability study. The test-retest reliability of the cervical vertebra activity data was analyzed using ICC, SEM and MDC (Table I). The data revealed that the CROM and OMCS 


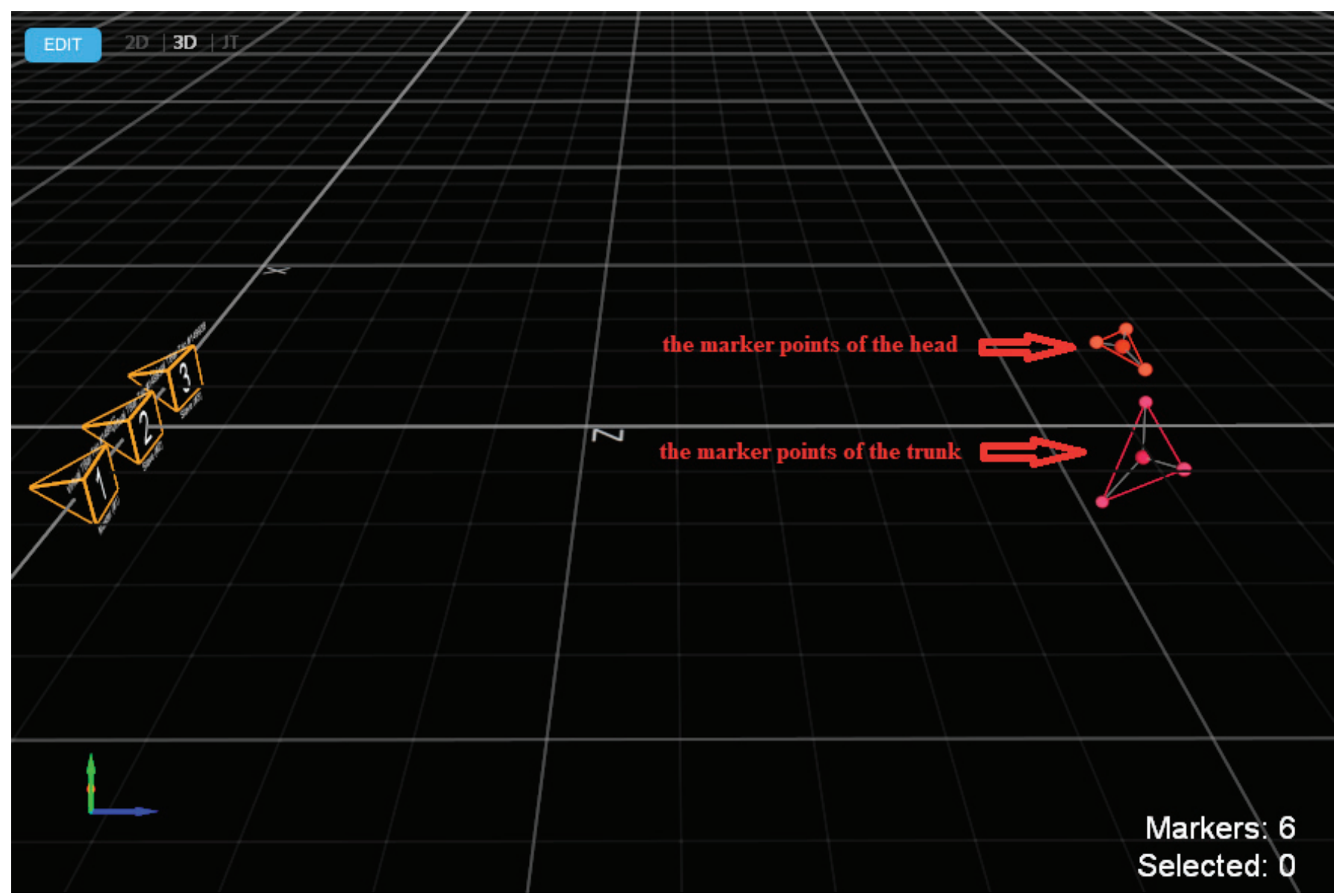

Figure 3. Six marker points on the torso are visualized using Optitrack Motive software. Numbers 1, 2 and 3 represent the Optitrack cameras.

methods had a good repeatability. All ICCs were at the substantial or almost perfect level [CROM device, ICC $(1,2)$ =0.768-0.948; OMCS, ICC $(1,2)=0.802-0.981$; Table I] . CROM and OMCS methods had the highest reliability when the cervical spine was in extension. The lowest reliability was obtained when the right lateral bending of the cervical vertebrae was measured. Changes in the SEM and MDC values of CROM and OMCS methods were small for all neck ROM directions. SEM values range from $1.04^{\circ}$ (right lateral bending) to $2.45^{\circ}$ (extension) using the CROM device and from $0.98^{\circ}$ (right lateral bending) to $1.38^{\circ}$ (flexion) using the OMCS method. The MDC values were between $2.89^{\circ}$ (right lateral bending) and $6.78^{\circ}$ (extension) for the CROM device and between $2.72^{\circ}$ (right lateral bending) and $3.81^{\circ}$ (flexion) for the OMCS (Table I). The OMCS measured all cervical ROMs at a higher ICC value compared with the CROM device. The SEM and MDC ranges were also lower in the OMCS devise, indicating that both exhibit good repeatability. However, the results demonstrated that the repeatability of OMCS was more reliable compared with the CROM device.

Validity study. As presented in Table II, comparisons of the cervical ROM measurements were recorded using the OMCS and CROM devices. The results revealed that the mean difference varied little between them $(0.81 \pm 1.47$, right lateral bending; $3.39 \pm 3.04$, right rotation). Pearson's correlation coefficient for the cervical movements was between 0.753 and 0.939 (Table II), indicating a significant correlation. The

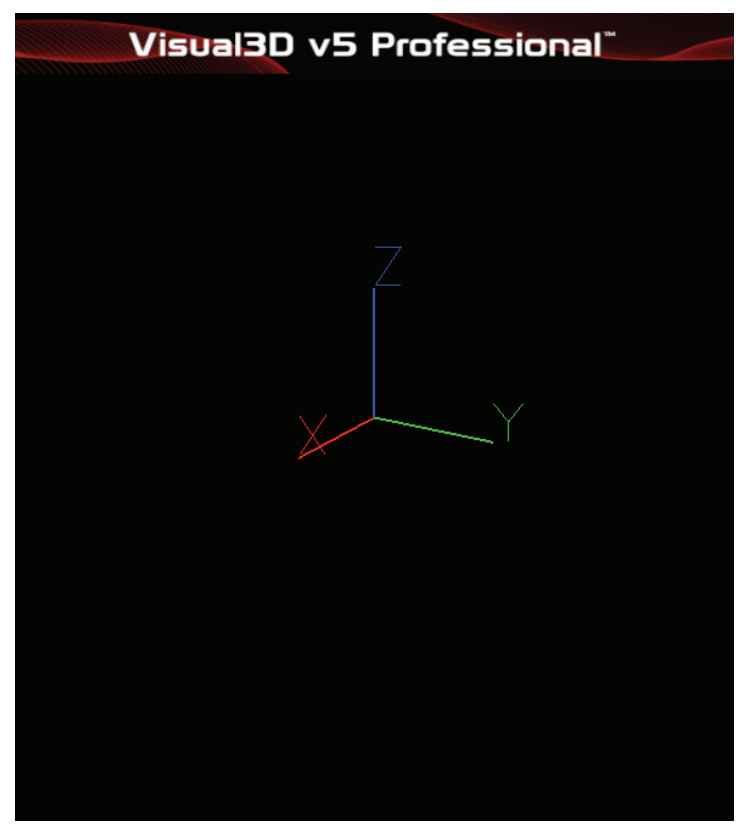

Figure 4. Movement track of the OptiTrack Motive software record was modeled and angles were calculated using the Visual3D software.

results of the regression analysis for all movements revealed a high $\mathrm{R}^{2}$ (0.568-0.882). Fig. 6 presents the regression lines and scatter plots for each movement. Bland-Altman plots of all movements reveal that differences were scattered close to zero, 


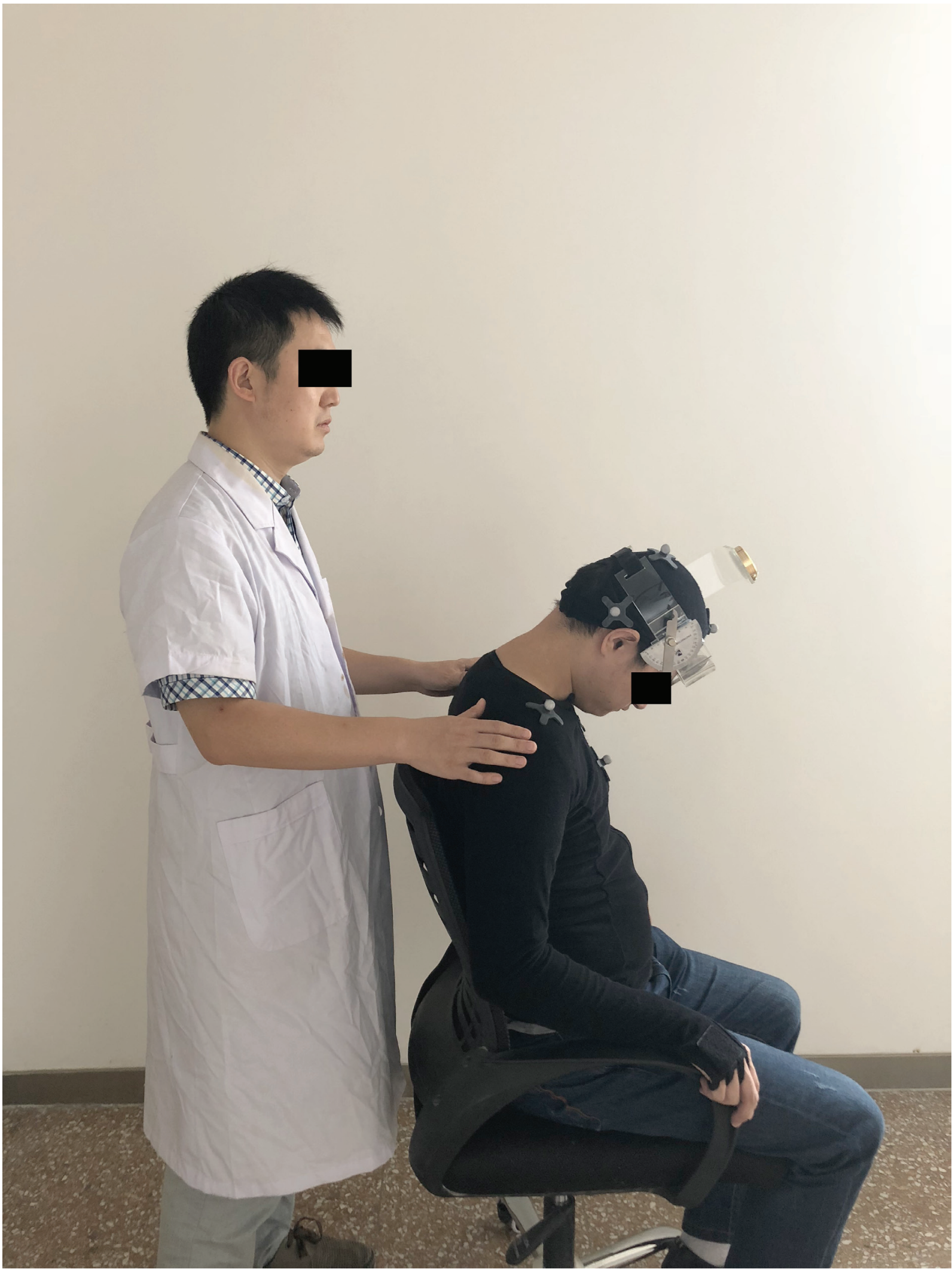

Figure 5. Measurement of cervical motion. A physician, with his hands placed on the shoulders of the participant, assists in bodily fixation.

with the majority of data points located within the $95 \% \mathrm{CI}$ (Fig. 7). All determined correlation coefficients, demonstrate that OMCS and CROM measurements are in good agreement, indicating that OMCS is an effective technique for ROM measurement of the cervical vertebrae.

\section{Discussion}

A variety of neck diseases, such as cervical spondylosis, directly cause limited cervical vertebrae mobility (6). The degree of cervical vertebral ROM reduction is associated with the 
severity of neck disease (26). The American College of Physicians use cervical ROM as a standard for determining the level of cervical vertebral damage (27). The accurate measurement of cervical vertebral ROM is therefore important in clinical practice. A variety of methods are used to measure cervical spine mobility. A visual measurement is often used as it has the advantage of being simple and repeatable, although the error range is large (9). A tape measure can be used to measure changes in anatomical landmarks on the body surface during cervical spine activity and indirectly reflecting the ROM of the cervical vertebra (10). Whitcroft et al (17) demonstrated that tape measurements were not completely accurate when compared with measurements obtained using a CROM device. Therefore, the authors determined that tape measurements are inaccurate and should not be used in clinical practice. However, more evidence-based medical studies are required to clarify the reliability and validity of tape measurements for cervical spine ROM. Physicians often use either a visual survey (8) or a CROM device (3) to determine neck ROM clinically and in formal studies. The torso however inevitably moves in conjunction with neck movement, so cervical ROM results include the combined movement of the trunk and neck (28). Additionally, when measuring the mobility of the neck in one direction, it should be considered that neck motions involve more than one plane (29). Within observations of cervical flexion, the cervical vertebrae exhibit lateral flexion and rotational direction motion.

According to the results of the current study, the OMCS obtains values of higher accuracy when measuring cervical ROM. OMCS measures three-dimensional motions dynamically and accurately and its technology eliminates the influence of trunk activity via software calculations of cervical vertebral activity. Therefore, OMCS only calculates the angle of cervical ROM. Using visual 3D software, the three-dimensional angle change in cervical vertebral activity can be accurately calculated, without the use of the plane angle.

In the present study, OMCS was used to detect cervical vertebral activity. The results were: Cervical flexion $\left(63.31 \pm 7.35^{\circ}\right)$, extension $\left(62.15 \pm 9.85^{\circ}\right)$, left lateral bending $\left(52.87 \pm 3.03^{\circ}\right)$, right lateral bending $\left(49.17 \pm 2.21^{\circ}\right)$, left rotation $\left(81.2 \pm 3.05^{\circ}\right)$ and right rotation $\left(79.36 \pm 3.77^{\circ}\right)$. With the exception of flexion, which may be due to this being the first action, these ROM values were lower than those obtained with the CROM device. The participant torso was close to the back of the chair to reduce involuntary torso movements when the actions were performed. However, the CROM device measured the coupled angle of the neck and trunk to account for this. The current study demonstrated that OMCS measurements of cervical vertebra had a high ICC and low SEM and MDC values when compared with those obtained using the CROM device. These results are consistent with the results obtained by Inokuchi et al (28). This consistency may be due to the increased accuracy of OMCS as it is capable of capturing fast moving objects with a global shutter imager and 120 FPS capture speed. Using advanced image processing to maximize resolution from three 640x480 VGA sensors, the OMCS can also track markers down to sub-millimeter movements with repeatable accuracy (Available from: www.optitrack.com/ products/v120-trio/specs.html). There may be subjective bias when using CROM. The CROM device was selected for the 
Table II. Validity of the two methods used to measure cervical ROM.

\begin{tabular}{|c|c|c|c|c|c|}
\hline \multirow{2}{*}{ Movement } & \multicolumn{2}{|c|}{ Cervical ROM SD $(\mathrm{x} \pm \mathrm{s})$} & \multirow{2}{*}{$\begin{array}{c}\text { Mean } \\
\text { difference }(x \pm s)\end{array}$} & \multirow{2}{*}{$\begin{array}{l}\text { Pearson's correlation } \\
\text { coefficients }\end{array}$} & \multirow{2}{*}{$\begin{array}{l}\text { Determination } \\
\text { coefficient }\end{array}$} \\
\hline & OMCS & CROM & & & \\
\hline Flexion & $63.31 \pm 7.35$ & $60.15 \pm 8.46$ & $-3.16 \pm 2.96$ & 0.94 & 0.88 \\
\hline Extension & $62.15 \pm 9.85$ & $64.75 \pm 10.73$ & $2.6 \pm 4.43$ & 0.91 & 0.83 \\
\hline Left lateral bending & $52.87 \pm 3.03$ & $54.4 \pm 4.56$ & $1.53 \pm 2.88$ & 0.79 & 0.62 \\
\hline Right lateral bending & $49.17 \pm 2.21$ & $49.98 \pm 2.17$ & $0.81 \pm 1.47$ & 0.79 & 0.63 \\
\hline Left rotation & $81.2 \pm 3.05$ & $82.1 \pm 2.26$ & $0.9 \pm 2.10$ & 0.77 & 0.59 \\
\hline Right rotation & $79.36 \pm 3.77$ & $82.65 \pm 4.19$ & $3.39 \pm 3.04$ & 0.75 & 0.57 \\
\hline
\end{tabular}

ROM, range of motion; CROM, cervical range of motion; OMCS, optical motion capture system.
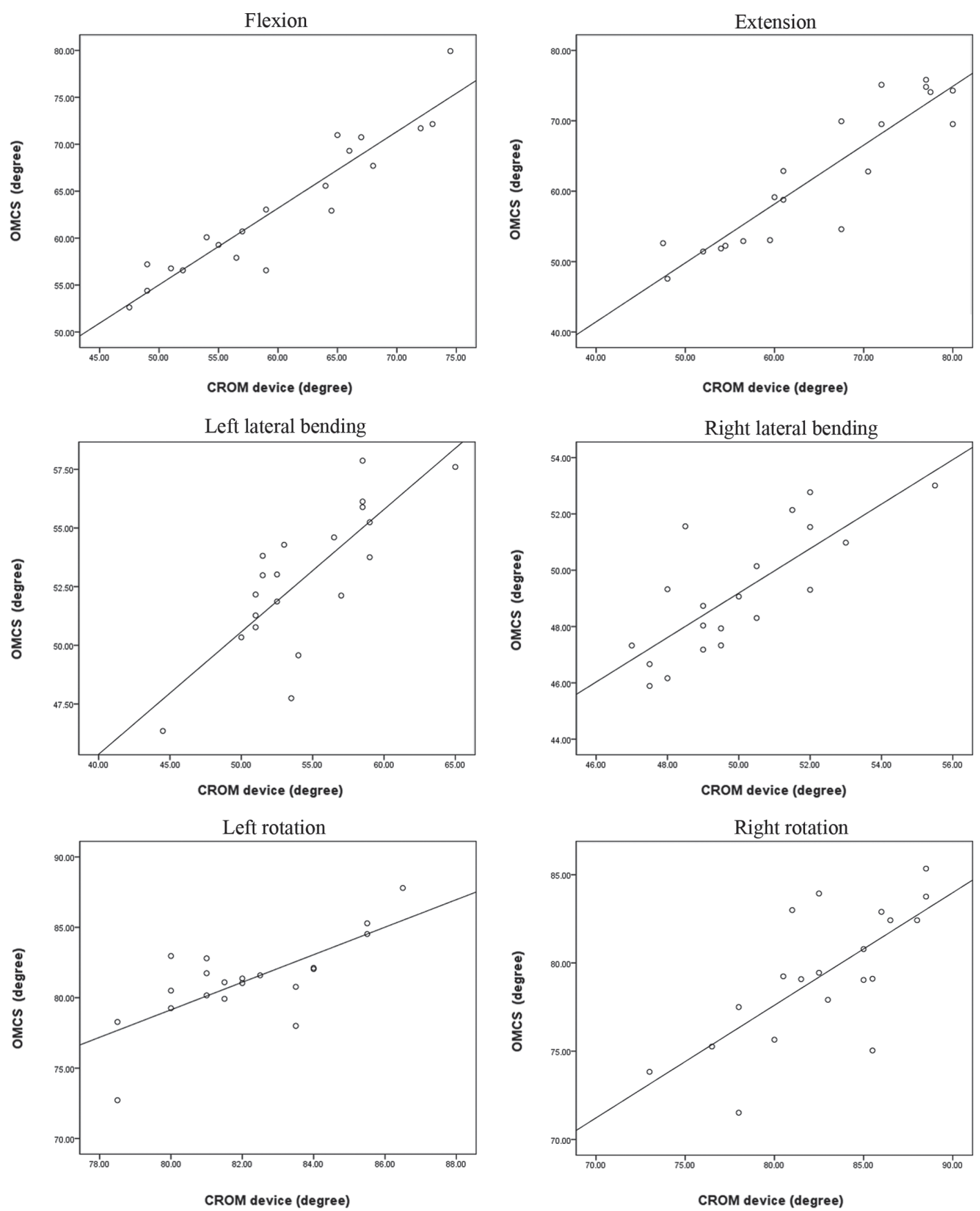

Figure 6. Regression analysis of the CROM measured by the optical motion capture system and the CROM device. CROM, cervical range of motion. 

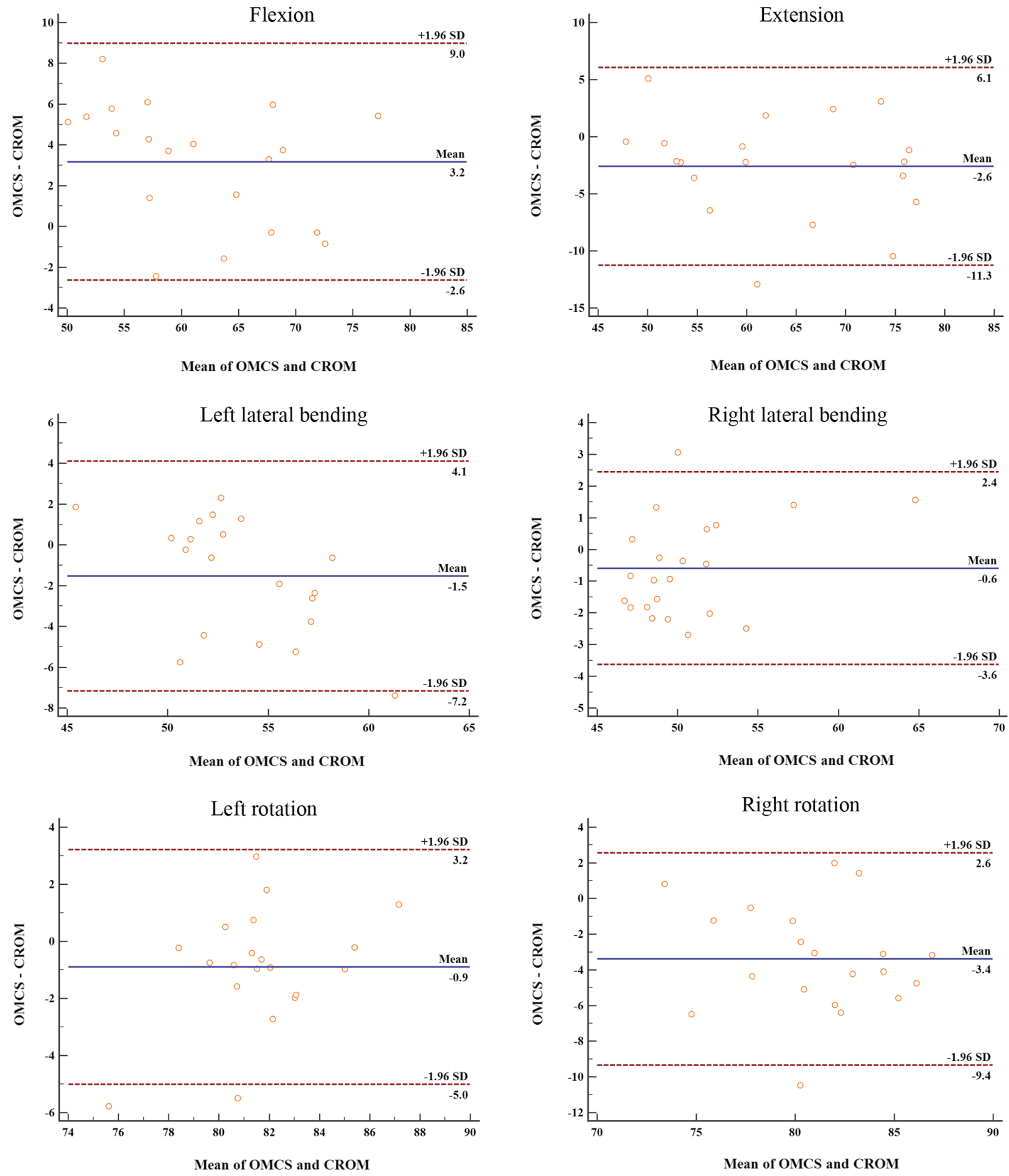

Figure 7. Bland-Altman plots of the mean of the OMCS and CROM measurements. CROM, cervical range of motion.

present study due to it being previously tested, revealing it to exhibit high validity and repeatability for the measurement of cervical ROM $(2,18,19)$. CROM has also been used in other studies $(13,30)$. Neck muscle strength is associated with cervical vertebrae activity (31), therefore the decrease of muscle strength may cause the decrease of cervical ROM. Healthy individuals were selected as participants in order decrease variability between participants and reduce bias.

The results of the present study indicated that CROM and OMCS techniques exhibit advantages and disadvantages. OMCS exhibits high accuracy and repeatability when measuring cervical vertebral ROM. However, this technology requires high quality equipment, as well as physicians that are familiar with the equipment and software operations. OMCS disadvantages include the complicated operation and long data processing times. OMCS may therefore be suitable for scientific research that requires accurate calculations and measurements of neck ROM. In contrast, the CROM device offers a convenient operation, low price and immediate results. However, the measurement of error is slightly larger, which is acceptable for measuring cervical vertebral ROM in patients with cervical diseases, clinically (2). 
The age range and sample size of the participants in the current study is a limiting factor; the present study is a preliminary investigation into the validity of using the OMCS and CROM methods. A total of 20 healthy young participants were enrolled in the current study. Individual differences in the neck may exist. Future studies should therefore focus on the inclusion a greater number of patients of a variety of ages with neck disorders. Finally, due to all participants wearing the same cat suit with fixed marker points, the slack that developed in the cat suit over time caused the marker points to change position, resulting in measurement errors in addition to those due to individual fat- and lean-tissue differences.

The results of the current study confirmed the high repeatability and validity of neck ROM measurements acquired using OMCS. This device can therefore be recommended for measuring cervical ROM for research purposes. Future studies that include patient groups with cervical diseases should be conducted to further substantiate the results of the current study.

\section{Acknowledgements}

Not applicable.

\section{Funding}

No funding was received.

\section{Availability of data and materials}

All data generated or analyzed during this study are included in this published article.

\section{Authors' contributions}

LZ contributed to the conception and design of the current study. LL, MF, WS and GL designed and performed the experiments. TH, XY and XW recorded and analyzed the operation-related data. LL and MF wrote and revised the manuscript. All authors read and approved the final manuscript.

\section{Ethics approval and consent to participate}

The current study was approved by the Ethics Committee of Wangjing Hospital of Chinese Academy of Chinese Medical Sciences (Beijing, China; institutional review board reference no. WJEC-KT-2017-020-P001). Signed informed consents were obtained from the individuals who participated in the current study.

\section{Patient consent for publication}

Signed informed consents were obtained from the individuals who participated in the current study.

\section{Competing interests}

The authors declare that they have no competing interests.

\section{References}

1. Cohen SP and Hooten WM: Advances in the diagnosis and management of neck pain. BMJ 358: j3221, 2017.

2. Côté P, Cassidy JD, Carroll LJ and Kristman V: The annual incidence and course of neck pain in the general population: A population-based cohort study. Pain 112: 267-273, 2004.

3. Audette I, Dumas JP, Cote JN and De Serres SJ: Validity and between-day reliability of the cervical range of motion (CROM) device. J Orthop Sports Phy Ther 40: 318-323, 2010.

4. Aranha MF, Muller CE and Gaviao MB: Pain intensity and cervical range of motion in women with myofascial pain treated with acupuncture and electroacupuncture: A double-blinded, randomized clinical trial. Braz J Phy Ther 19: 34-43, 2015.

5. Silva ACO, Biasotto-Gonzalez DA, Oliveira FHM, Andrade AO, Gomes CAFP, Lanza FC, Amorim CF and Politti F: Effect of osteopathic visceral manipulation on pain, cervical range of motion, and upper trapezius muscle activity in patients with chronic nonspecific neck pain and functional dyspepsia: A randomized, double-blind, placebo-controlled pilot study. Evid Based Complement Alternat Med 2018: 4929271, 2018.

6. Gao Z, Song H, Ren F, Li Y, Wang D and He X: Reliability and validity of CODA motion analysis system for measuring cervical range of motion in patients with cervical spondylosis and anterior cervical fusion. Exp Ther Med 14: 5371-5378, 2017.

7. Bogduk N and Mercer S: Biomechanics of the cervical spine. I: Normal kinematics. Clin Biomech (Bristol, Avon) 15: 633-648, 2000.

8. Kubas C, Chen YW, Echeverri S, McCann SL, Denhoed MJ, Walker CJ, Kennedy CN and Reid WD: Reliability and validity of cervical range of motion and muscle strength testing. J Strength Cond Res 31: 1087-1096, 2017.

9. Hirsch BP, Webb ML, Bohl DD, Fu M, Buerba RA, Gruskay JA and Grauer JN: Improving visual estimates of cervical spine range of motion. Am J Orthop (Belle Mead NJ) 43: E261-E265, 2014.

10. Asha SE and Pryor R: Validation of a method to assess range of motion of the cervical spine using a tape measure. J Manipulative Physiol Ther 36: 538-545, 2013.

11. Dunleavy K, Neil J, Tallon A and Adamo DE: Reliability and validity of cervical position measurements in individuals with and without chronic neck pain. J Man Manip Ther 23: 188-196, 2015.

12. De-la-Puente-Ranea L, Garcia-Calvo B, La Touche R, Fernandez-Carnero J and Gil-Martinez A: Influence of the actions observed on cervical motion in patients with chronic neck pain: A pilot study. J Exerc Rehabil 12: 346-354, 2016.

13. Wibault J, Vaillant J, Vuillerme N, Dedering A and Peolsson A: Using the cervical range of motion (CROM) device to assess head repositioning accuracy in individuals with cervical radiculopathy in comparison to neck- healthy individuals. Man Ther 18: 403-409, 2013.

14. Tousignant M, de Bellefeuille L, O'Donoughue S and Grahovac S: Criterion validity of the cervical range of motion (CROM) goniometer for cervical flexion and extension. Spine (Phila Pa 1976) 25: 324-330, 2000.

15. Ito K, Yukawa Y, Machino M, Kobayakawa A and Kato F: Range of motion determined by multidetector-row computed tomography in patients with cervical ossification of the posterior longitudinal ligament. Nagoya J Med Sci 77: 221-228, 2015.

16. Maksymowych WP, Mallon C, Richardson R, Conner-Spady B, Jauregui E, Chung C, Zappala L, Pile K and Russell AS: Development and validation of a simple tape-based measurement tool for recording cervical rotation in patients with ankylosing spondylitis: Comparison with a goniometer-based approach. J Rheumatol 33: 2242-2249, 2006.

17. Whitcroft KL, Massouh L, Amirfeyz R and Bannister G: Comparison of methods of measuring active cervical range of motion. Spine (Phila Pa 1976) 35: E976-E980, 2010.

18. Tousignant M, Smeesters C, Breton AM, Breton E and Corriveau H: Criterion validity study of the cervical range of motion (CROM) device for rotational range of motion on healthy adults. J Orthop Sports Phys Ther 36: 242-248, 2006.

19. Tousignant $M$, Duclos E, Lafleche S, Mayer A, Tousignant-Laflamme Y, Brosseau L and O'Sullivan JP: Validity study for the cervical range of motion device used for lateral flexion in patients with neck pain. Spine (Phila Pa 1976) 27: 812-817, 2002.

20. Aurand AM, Dufour JS and Marras WS: Accuracy map of an optical motion capture system with 42 or 21 cameras in a large measurement volume. J Biomech 58: 237-240, 2017. 
21. Stratford PW and Goldsmith $\mathrm{CH}$ : Use of the standard error as a reliability index of interest: An applied example using elbow flexor strength data. Phys Ther 77: 745-750, 1997.

22. Beaton DE, Bombardier C, Katz JN and Wright JG: A taxonomy for responsiveness. J Clin Epidemiol 54: 1204-1217, 2001

23. Landis JR and Koch GG: The measurement of observer agreement for categorical data. Biometrics 33: 159-174, 1977.

24. Mehta S, Bastero-Caballero RF, Sun Y, Zhu R, Murphy DK, Hardas B and Koch G: Performance of intraclass correlation coefficient (ICC) as a reliability index under various distributions in scale reliability studies. Stat Med 37: 2734-2752, 2018.

25. Bland JM and Altman DG: Agreed statistics: Measurement method comparison. Anesthesiology 116: 182-185, 2012.

26. Langenfeld A, Bastiaenen C, Sieben J and Swanenburg J: Development and validation of a self-administered neck mobility assessment tool (S-ROM-Neck) in chronic neck pain patients. Musculoskel Sci Pract 37: 75-79, 2018.

27. Dvir Z, Gal-Eshel N, Shamir B, Prushansky T, Pevzner E and Peretz C: Cervical motion in patients with chronic disorders of the cervical spine: A reproducibility study. Spine (Phila $\mathrm{Pa}$ 1976) 31: E394-E399, 2006.
28. Inokuchi $\mathrm{H}$, Tojima $\mathrm{M}$, Mano $\mathrm{H}$, Ishikawa $\mathrm{Y}$, Ogata $\mathrm{N}$ and Haga N: Neck range of motion measurements using a new three-dimensional motion analysis system: Validity and repeatability. Eur Spine J 24: 2807-2815, 2015

29. Song H, Li HP, Gao ZY, Gao ZC and He XJ: The research status and progress of cervical range motion measurement. Orthopaedic Biomechanics Materials and Clinical Study 14: 63-69, 2017 (In Chinese)

30. Williams MA, Williamson E, Gates S and Cooke MW Reproducibility of the cervical range of motion (CROM) device for individuals with sub-acute whiplash associated disorders. Eur Spine J 21: 872-878, 2012.

31. Lee H, Nicholson LL and Adams RD: Cervical range of motion associations with subclinical neck pain. Spine 29: 33-40, 2004.

This work is licensed under a Creative Commons Attribution-NonCommercial-NoDerivatives 4.0 International (CC BY-NC-ND 4.0) License. 